\title{
Damage Diagnosis of Rotors: Application of Hilbert Transform and Multi-Hypothesis Testing
}

\author{
MICHAEL FELDMAN \\ Faculty of Mechanical Engineering, \\ Technion - Israel Institute of Technology, \\ Haifa 32000, Israel \\ SUSANNE SEIBOLD \\ Institut für Techno- und Wirtschaftsmathematik, \\ Erwin-Schrödinger-Straße, \\ D-67663 Kaiserslautern, Germany
}

\begin{abstract}
In this paper, a combined approach to damage diagnosis of rotors is proposed. The intention is to employ signal-based as well as model-based procedures for an improved detection of size and location of the damage. In a first step, Hilbert transform signal processing techniques allow for a computation of the signal envelope and the instantaneous frequency, so that various types of non-linearities due to a damage may be identified and classified based on measured response data. In a second step, a multi-hypothesis bank of Kalman Filters is employed for the detection of the size and location of the damage based on the information of the type of damage provided by the results of the Hilbert transform.
\end{abstract}

Keywords: Hilbert transform, damage diagnosis, Kalman filtering, non-linear dynamics

\section{INTRODUCTION}

Modern machinery is bound to fulfill increasing demands concerning durability as well as safety requirements. In order to manage these complex tasks, procedures for an automated damage detection have to be provided. Current trends are described e.g. by Williams and Davies (1992). The diagnosis of turbomachinery is of particular relevance, in order to avoid catastrophic damage or injury, Haas (1977), Muszynska (1992). Basically, one can distinguish between signal-based approaches, which merely rely on an analysis of the measurements, and model-based approaches, which additionally utilize an appropriate model of the system under investigation.

Signal-based diagnosis employs our physical understanding of the dynamic system behavior in the presence of a specific damage. For instance, a crack in a rotor shaft influences the rotor vibrations, especially the first, second and third harmonics. Besides, a shifting of the phase occurs. Therefore, a suitable analysis of the measurement signals yields valuable hints for the detection of a crack. Furthermore, non-linearities due to the "breathing" (the opening and closing of the crack) are introduced.

In the recent past the characterization of the response of non-linear vibration systems has been approached using the Hilbert transform (HT) in the time domain. The objective was to propose a methodology to identify and classify various types of non-linearities from measured response data, Feldman (1994a, 1994b). The proposed methodology also concentrated on the HT signal processing techniques, which allow for the computation of the signal envelope and the instantaneous frequency. It was shown that the system instantaneous dynamic parameters and also the elastic non-symmetric force characteristics can be estimated, Feldman (1997). The results showed that the methodology is very well applicable to the diagnosis of damage, too.

In general, the drawback of the signal-based procedures is that most of the time the determination of location and extent of a damage is not possible. This gap can be filled by model-based procedures, where a numerical 
relation between the damage and a specific damage parameter is established. The detection is performed by relating algorithmically a model of the system under investigation to the measurements. A suitable analysis of the differences between model and measurements yields the determination of the location and size of the damage. For instance, a bank of Kalman Filters may be employed for the detection of the location of a damage like a crack in a rotor shaft. Furthermore, its depth may be calculated, too, Seibold and Weinert (1996).

However, this model-based approach is quite time-consuming and usually not applicable for on-line monitoring. Furthermore, it might sometimes be difficult to distinguish between specific damages like e.g. a crack and an increasing unbalance. Therefore, we propose to combine the two methods for an improved detectability of damage at early stages: the HT signal processing technique will be employed for a characterization of the damage, so that in a second step, a bank of Kalman Filters based on a suitable model of the damage can be designed. Then, the location and extent of the damage can be calculated.

The paper is organized according to the following: Section 2 focuses on the HT. First, the theoretical background is explained. Then, a new approach for the application to non-linear systems is discussed. Section 3 aims at clarifying the procedure with a simulation study. In section 4, some basic ideas for the modeling of a crack in a rotor shaft are presented, and a finite crack model is described. Section 6 briefly discusses the idea of multi-hypothesis testing based on a bank of Kalman Filters. Finally, in section 6, the methods presented are applied for the diagnosis of a crack in the shaft of a rotor test rig. Section 7 discusses the results. 


\section{THEORETICAL BACKGROUND: THE HILBERT TRANSFORM}

The considered HT identification methods in the time domain have been developed by one of the authors for only symmetrical non-linearities, Feldman $(1985,1994 a)$. As only symmetrical non-linearities were considered, non-linear elastic and damping force characteristic can be expressed in terms of odd polynomials. However, it is also possible to extend the method in order to analyze vibration systems with non-symmetrical non-linearities. The main idea of the method is to take the solution of the non-symmetrical vibration system in the time domain and split it into two different "subsolutions" separately for the positive and for the negative displacement. Then, the separated solutions of the non-symmetric system are determined by adopting the method developed for the non-linear vibration system identification, Feldman $(1985,1994 a)$.

\subsection{Non-Linear System Representation}

The equation of free vibration of a non-linear non-symmetrical structure can be written as follows:

$$
\begin{gathered}
\ddot{y}+2 h \dot{y}+F(y)=0 \\
F(y)=\left\{\begin{array}{l}
F_{1}(y), \text { if } y>0 \\
F_{2}(y), \text { if } y \leq 0
\end{array},\right.
\end{gathered}
$$

where $y$ - the system solution, $h$ - the damping coefficient, $F(y)$ - the non-linear non-symmetrical spring force, $F_{1}(y)$ - the non-linear stiffness characteristic for the positive displacement, $F_{2}(y)$ - the non-linear stiffness characteristic for the negative displacement. The solution of the equation depends mainly on the non-symmetrical elastic force $F(y)$, that can be expressed in terms of odd and even polynomials of displacement. In the case of positive displacement $y>0$ the solution is produced by the first line of the non-symmetrical elastic force characteristic, Figure 1. Each displacement sign changing from positive to negative, or the reverse, switches the vibration structure, which will include correspondingly the first or the second non-symmetrical elastic force characteristic. The oscillating force will be transformed into oscillatory motion with corresponding amplitude and frequency features. An illustration of non-symmetrical amplitude transformation of the solution is shown in Figure 1.

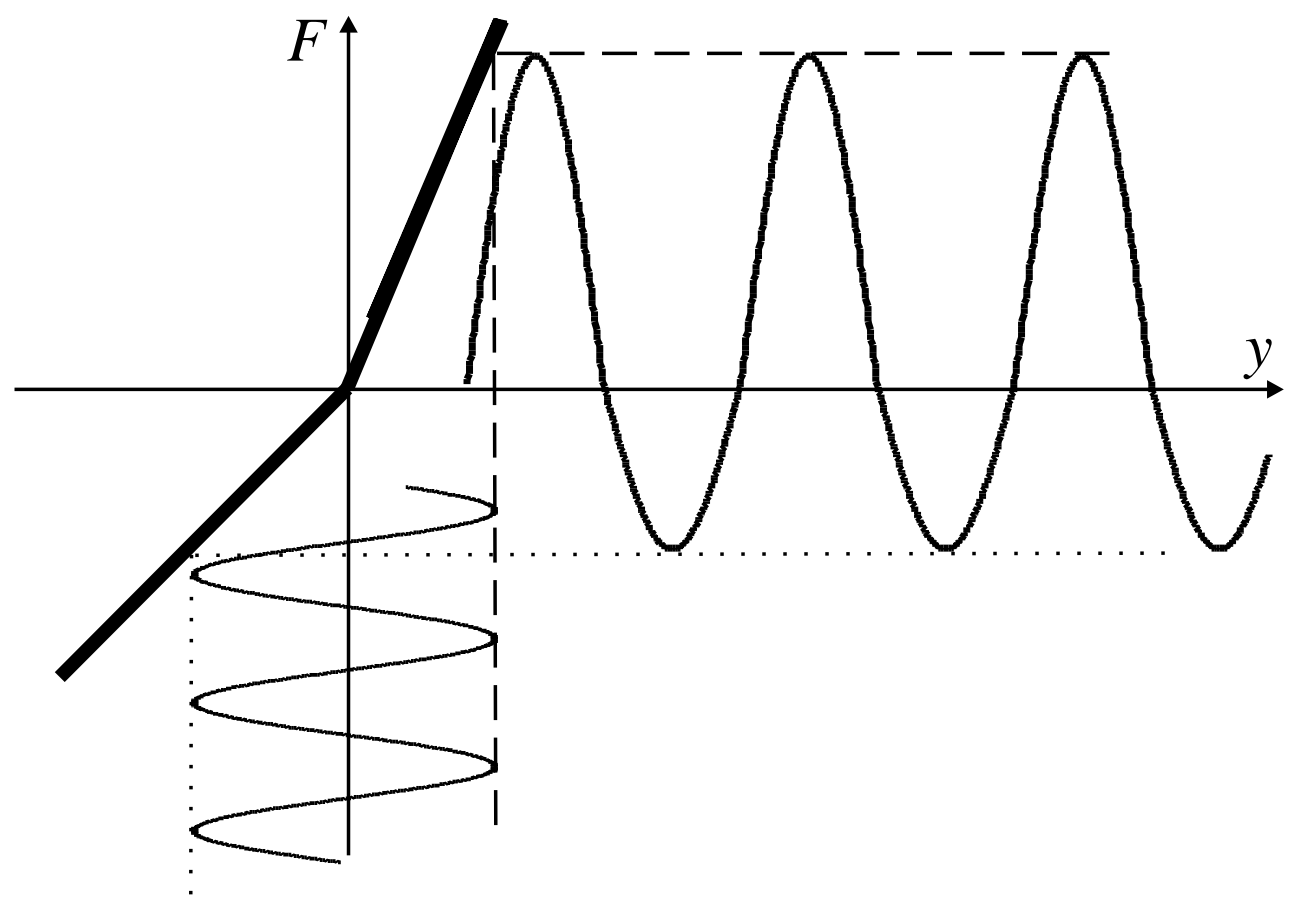

Figure 1: Illustration of transformation of amplitude of the vibration solution 
Suppose the solution of the system consists of two independent separate parts: a positive motion, associated only with the positive force characteristic curve, and a negative motion associated only with the negative force characteristic curve:

$$
y(t)=\left\{\begin{array}{l}
y_{1}(t), \text { if } y>0 \\
y_{2}(t), \text { if } y \leq 0
\end{array} .\right.
$$

The positive motion is only influenced by the positive force and conversely the negative motion is dependent upon the negative branch. In other words, according to this assumption each part of the system solution is determined only by its corresponded force characteristic. Each part of the vibration signal could be represented in the Analytic signal form: $y_{1,2}(t)=A_{1,2}(t) \cos \left[\int \omega_{1,2}(t)\right]$ where $y_{i}(t)$ - the vibration signal (real valued function), $A_{i}(t)$ - the envelope (instantaneous amplitude), $\omega_{i}(t)$ - the instantaneous frequency. One of the question arising immediately from this representation is: How will the combined signal $y(t)$ be separated into its constituent parts $y_{1}(t)$ and $y_{2}(t)$ ? The HT also could play an important role in the signal decomposition and may lead to practical results.

\subsection{Bi-Linear System Vibration Decomposition}

Originally consider the case of a vibrating conservative system with bi-linear force elastic characteristic $F_{1,2}=\omega_{1,2}^{2} y$. According to the assumption equation (2), the solution of the system is built up from two alternate harmonic. During a half of the period, when the displacement is positive, the vibration appears as a harmonic $A_{1} \cos \omega_{1} t$ and during the next half, when the displacement is negative, the vibration continues as another harmonic with the different amplitude and frequency $A_{2} \cos \omega_{2} t$. As a first approximation, assume that these harmonics have the same frequency and different amplitudes $\left(A_{1}>A_{2}\right)$. In this case, the signal can be modeled in the time domain as a monocomponent signal $y(t)$ with nonzero mean value $M$. That is,

$$
y(t)=A_{m} \cos \left(\omega_{m} t\right)+M,
$$

where $A_{m}=0.5\left(A_{1}+A_{2}\right)$ - the amplitude of harmonic, $M=0.5\left(A_{1}-A_{2}\right)$ - the mean value (offset) of the non-symmetric signal $y(t)$. According to the main properties of the HT, Mitra and Kaiser (1993), the Hilbert transformed projection $\tilde{y}(t)$ will not respond to the signal mean value

$$
\tilde{y}(t)=A_{m} \sin \left(\omega_{m} t\right)
$$

The two equations (3a) and (3b) will produce an analytic signal of the form:

$$
Y(t)=y(t)+j \tilde{y}(t)=A(t) \exp (j \omega t)
$$

where $A^{2}(t)=2 A_{m} M \cos \left(\omega_{m} t\right)+M^{2}+A_{m}^{2}{ }^{-}$the non-symmetric signal envelope squared, $\omega(t)=\omega_{m} \frac{A_{m} M \cos \left(\omega_{m} t\right)+A_{m}^{2}}{A^{2}(t)}-$ the non-symmetric signal instantaneous frequency.

From equation (4) it can be seen that the signal envelope consists of two different parts. There is a slowvarying part, which includes a sum of the amplitude squared together with the mean value squared, and also a fast varying (oscillating) part, which is the multiplication of the amplitude and the mean value with the function $\cos \left(\omega_{m} t\right)$. For such a case it is possible to separate the slow and the fast (oscillating) parts of a signal envelope (mentioned above) by using an ordinary filtration in the frequency domain. Thus only the fast part $A_{f}^{2}(t)$ will be retained after high pass filtration of the square of the signal envelope: 
$A_{f}^{2}(t)=2 A_{m} M \cos \left(\omega_{m} t\right)$. This new function is now just a monocomponent signal. Repeating application of the HT, the new envelope extraction is readily achieved: $\left|A_{f}^{2}\right|=2 A_{m} M$. Then, after an algebraic operation, we will get a simple formula for each non-symmetric amplitude estimation: $A_{1,2}=\sqrt{A^{2}-A_{f}^{2} \pm\left|A_{f}^{2}\right|}$, where $A_{1}$ - the positive amplitude, $A_{2}$ - the negative amplitude, $A$ - the envelope of the non-symmetric signal, $A_{f}$ - the fast oscillating part of a signal envelope, $\left|A_{f}^{2}\right|$ - the envelope (result) of the repeated application of the HT.

Let us consider the next approximation of a non-symmetric signal $y(t)$ as a combination of two harmonics with different frequencies $\omega_{1}, \omega_{2}$ and the same amplitude $A_{m}$. The difference between the positive and the negative harmonic will eventually produce a nonzero mean value of the signal $M_{\omega}$,

$$
y(t)=\left\{\begin{array}{l}
A_{m} \cos \omega_{1} t+M_{\omega}, \text { if } y>0 \\
A_{m} \cos \omega_{2} t+M_{\omega}, \text { if } y \leq 0
\end{array},\right.
$$

where $M_{\omega}=\frac{2 A_{m}}{\pi} \frac{\omega_{2}-\omega_{1}}{\omega_{2}+\omega_{1}}$ - the mean value of the non-symmetric signal. The instantaneous frequency of the signal will now be biased due to the nonzero mean value equation (4):

$$
\omega(t)=\left\{\begin{array}{l}
\omega_{1} b, \text { if } y>0 \\
\omega_{2} b, \text { if } y \leq 0
\end{array},\right.
$$

where $b=\left(A_{m} M_{\omega} \cos \omega_{m} t+A_{m}^{2}\right) /\left(A^{2}(t)\right)$. To simplify the last equation, we assume that the mean value $M_{\omega}$ of a non-symmetric signal is always less than the amplitude of the signal, $M_{\omega}<A_{m}$ :

$$
\omega(t)=\omega_{1,2}\left(1+\frac{M_{\omega}}{A_{m}} \cos \omega_{m} t\right) /\left(1+2 \frac{M_{\omega}}{A_{m}} \cos \omega_{m} t\right),
$$

where $\omega_{1,2}=\omega_{1}$ if $y>0 ; \omega_{1,2}=\omega_{2}$ if $y<0$. The obtained equation (7) explains that the instantaneous frequency of the non-symmetric cycle, associated with the lesser frequency, is slightly less than the initial frequency. Correspondingly, the instantaneous frequency associated with the larger frequency is moderately larger than the initial frequency. After using a separate lowpass filtration of the instantaneous frequency, associated with the positive and the negative portions of signal, we will get two biased frequencies of the non-symmetric signal (equation (5)): $\left\langle\omega_{\text {pos,neg }}\right\rangle=\omega_{1,2}\left(\left(1 \pm M_{\omega}^{2}\right) /\left(A_{m}^{2}\right)\right)$.

The obtained bias value is very small. For example, in extreme cases when $\omega_{2}=2 \omega_{1}$ the bias is only $10 \%$, suggesting that the bias value could be neglected. In consequence, the resultant formula for the component frequencies takes the form: $\omega_{1,2}=\left\langle\omega_{\text {pos,neg }}\right\rangle$. The use of the developed technique could result in a more precise estimation of both the amplitude and the frequency of non-symmetric vibration signals.

\subsection{The Hilbert Transform Identification Technique}

Vibration signal, suitable for the HT identification, should be a monocomponent signal derived from SDOF system directly, or obtained from a multi-DOF system after special decomposition or after band-pass filtration. This initial signal $y(t)=A(t) \cos \psi t$, where $y(t)$ - vibration signal (real valued function), $A(t)$ the envelope (instantaneous amplitude), $\psi(t)$ - the instantaneous phase, is assumed to be a free solution of a 
non-linear vibration system with frequency dependent damping $\ddot{y}+2 h(\dot{y}) \dot{y}+k(y) y=0$, where $2 h(\dot{y}) \dot{y}$ the non-linear viscous damping force characteristic, $k(y) y$ - the non-linear elastic force characteristic. It is desirable to recover non-linear backbones and damping curves from the measured vibration signal. For this purpose some resultant equations could be used, Feldman (1994a):

$$
\begin{aligned}
& \omega_{0}^{2}=\dot{\psi}^{2}-\frac{\ddot{A}}{A}+\frac{2 \dot{A}^{2}}{A^{2}}+\frac{\dot{A} \ddot{\psi}}{A \dot{\psi}} \\
& h(t)=-\frac{\dot{A}}{A}+\frac{\ddot{\psi}}{2 \dot{\psi}}
\end{aligned}
$$

where $\omega_{0}(\mathrm{t})$ - instantaneous undamped natural frequency of the system, $h(t)$ - instantaneous damping coefficient of the system, $\dot{\psi}, \mathrm{A}$ - instantaneous frequency and envelope (amplitude) of the vibration with their first and second derivatives. Algebraically equation (8) means that the HT identification method uses the initial displacement, velocity and also acceleration at a time. 


\section{APPLICATION OF THE HILBERT TRANSFORM TO NON-LINEAR SYSTEMS SIMULATION}

All simulation examples demonstrate the performance that can be achieved using the proposed technique. In order to focus our non-symmetric signal processing on the effects of the non-linear vibration systems identification, we also use the HT identification method, Feldman (1994a).

\subsection{Non-symmetric bi-linear free vibration}

Consider the initial simulated signal of free vibration of a non-symmetric bi-linear system:

$$
\begin{aligned}
& \ddot{y}+2 \dot{y}+F(y)=0 \\
& F(y)=\left\{\begin{array}{l}
(20 \pi)^{2} y, \text { if } y>0, \\
(40 \pi)^{2} y, \text { if } y \leq 0
\end{array}\right.
\end{aligned}
$$

which is shown in Figure 2 together with the separated envelopes. By applying the HT identification technique to the signal, backbones and damping curves shown in Figure 3 are obtained. In the case shown it is obvious that the natural frequencies are constant (correspondingly 10 and $20 \mathrm{~Hz}$ ), and the damping

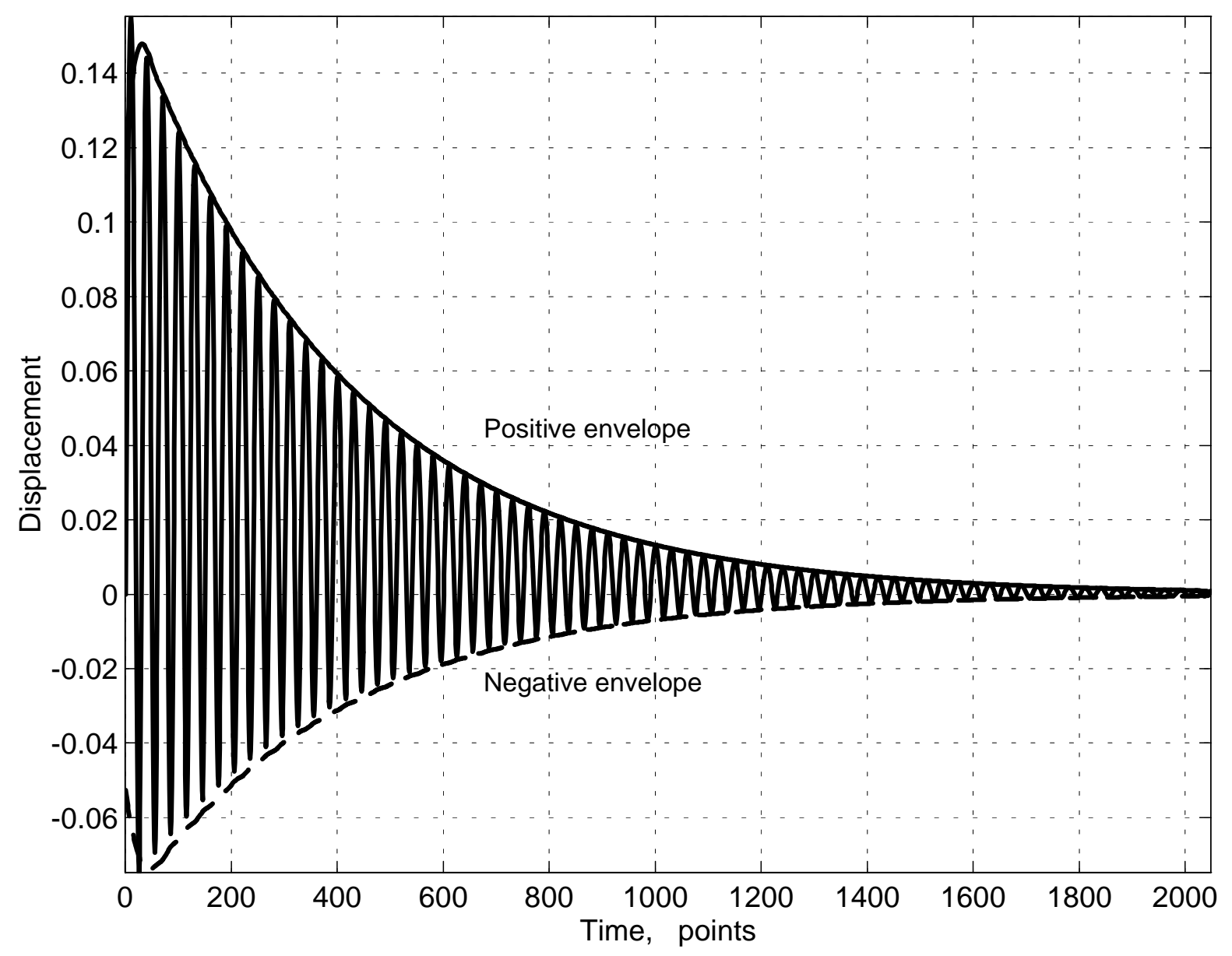

Figure 2: Bi-linear non-symmetric system free vibration 


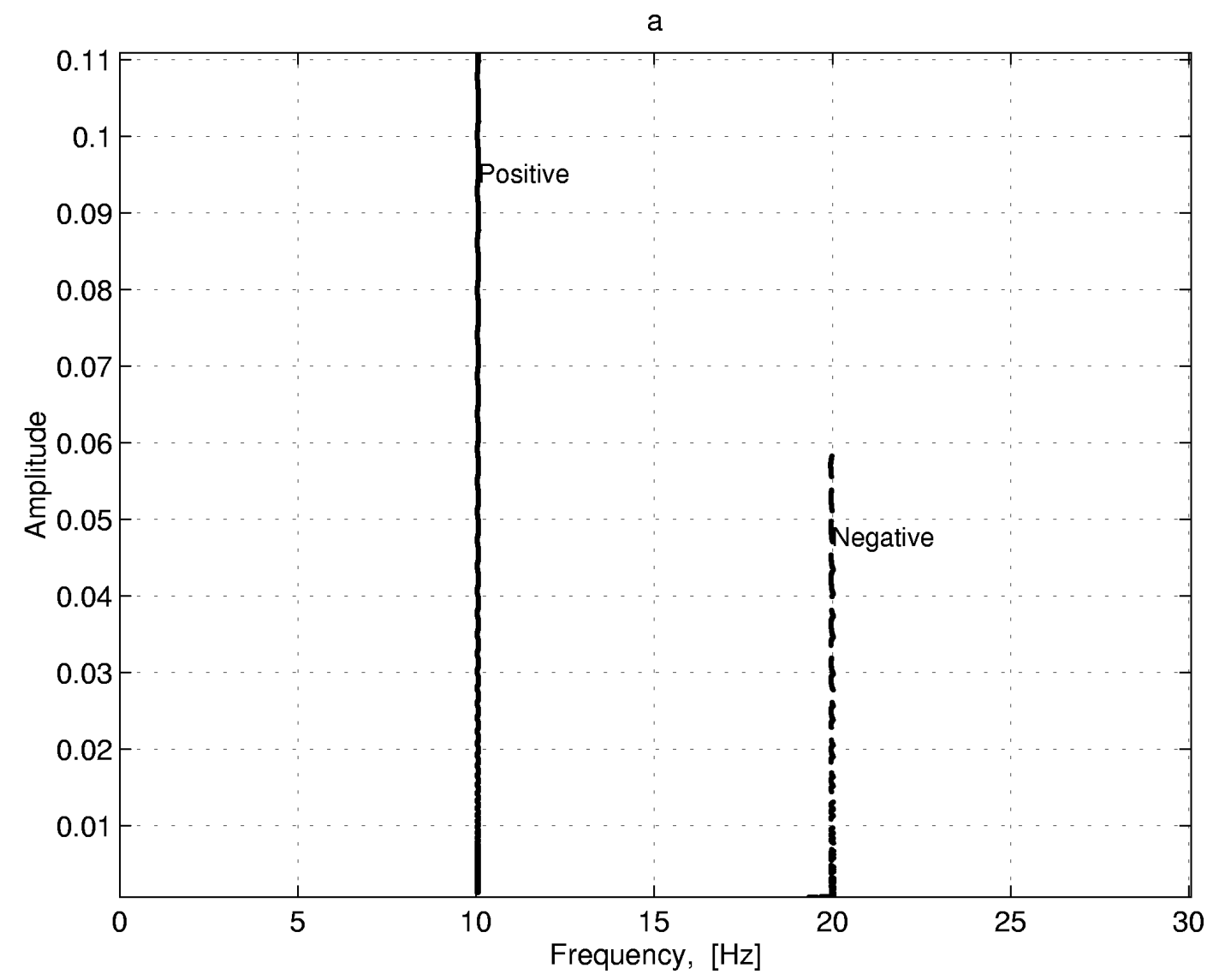

Figure 3a: Estimated force characteristics of bi-linear system - backbone

b

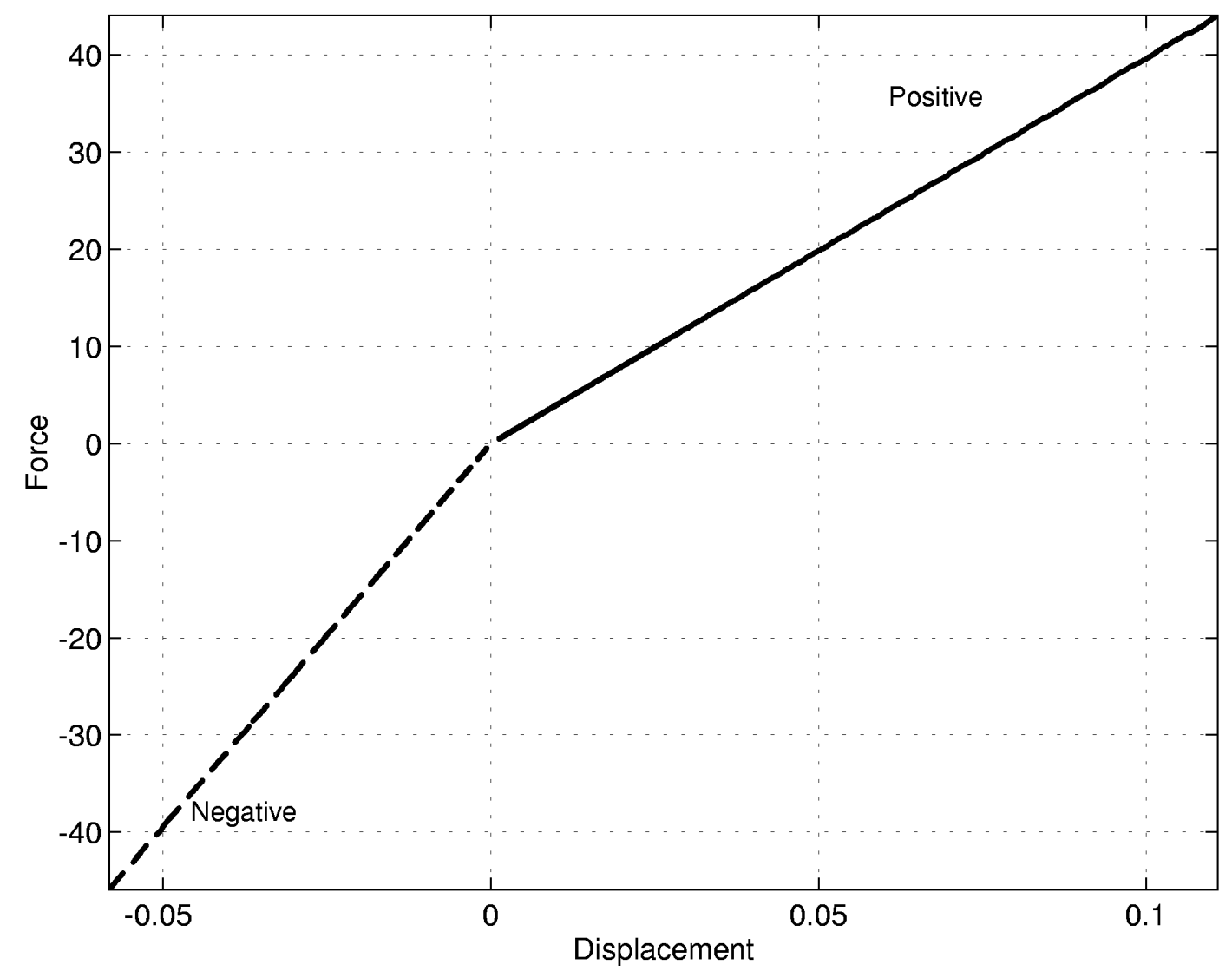

Figure 3b: Estimated force characteristics of bi-linear system - spring force characteristic 


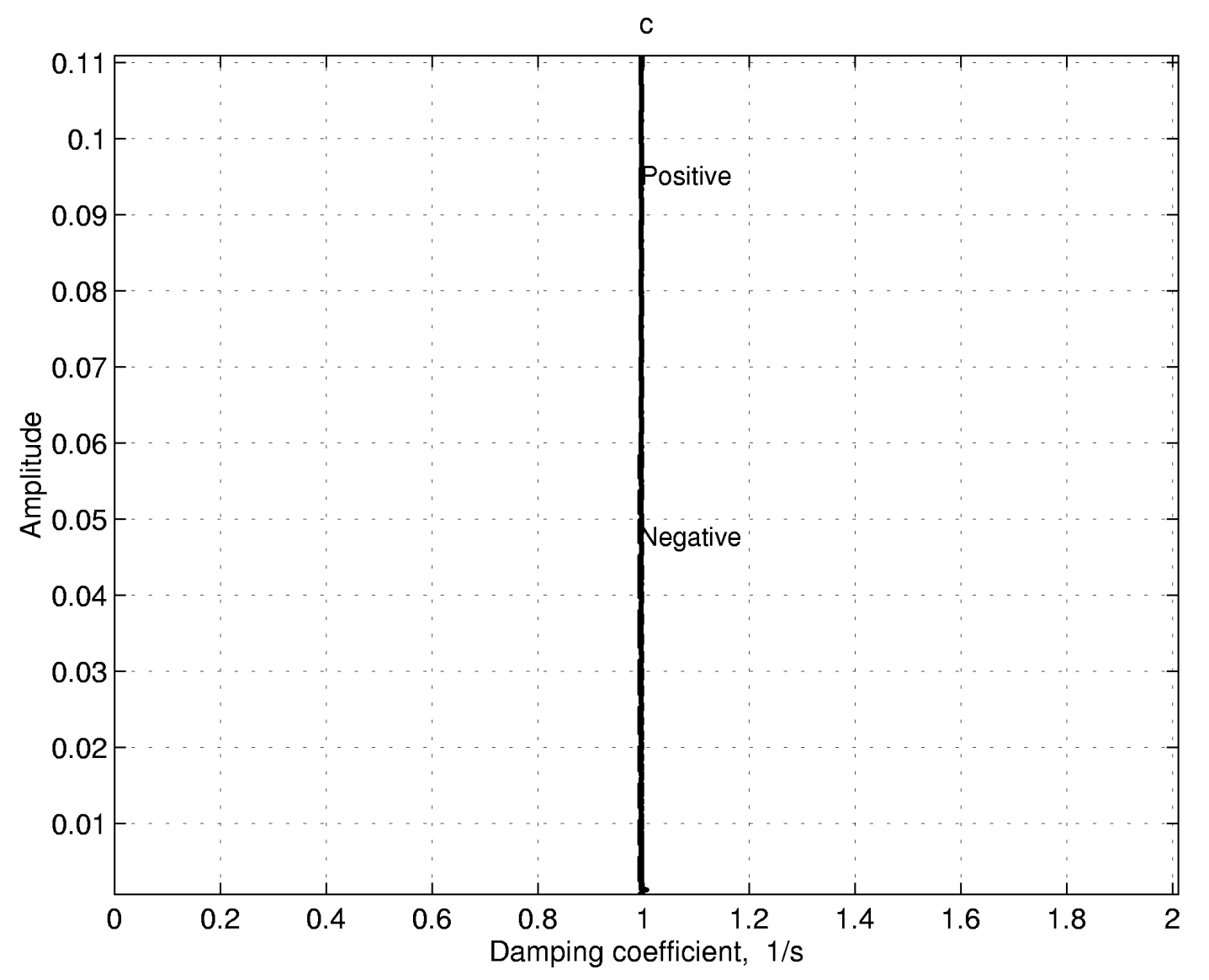

Figure 3c: Estimated force characteristics of bi-linear system - damping curve

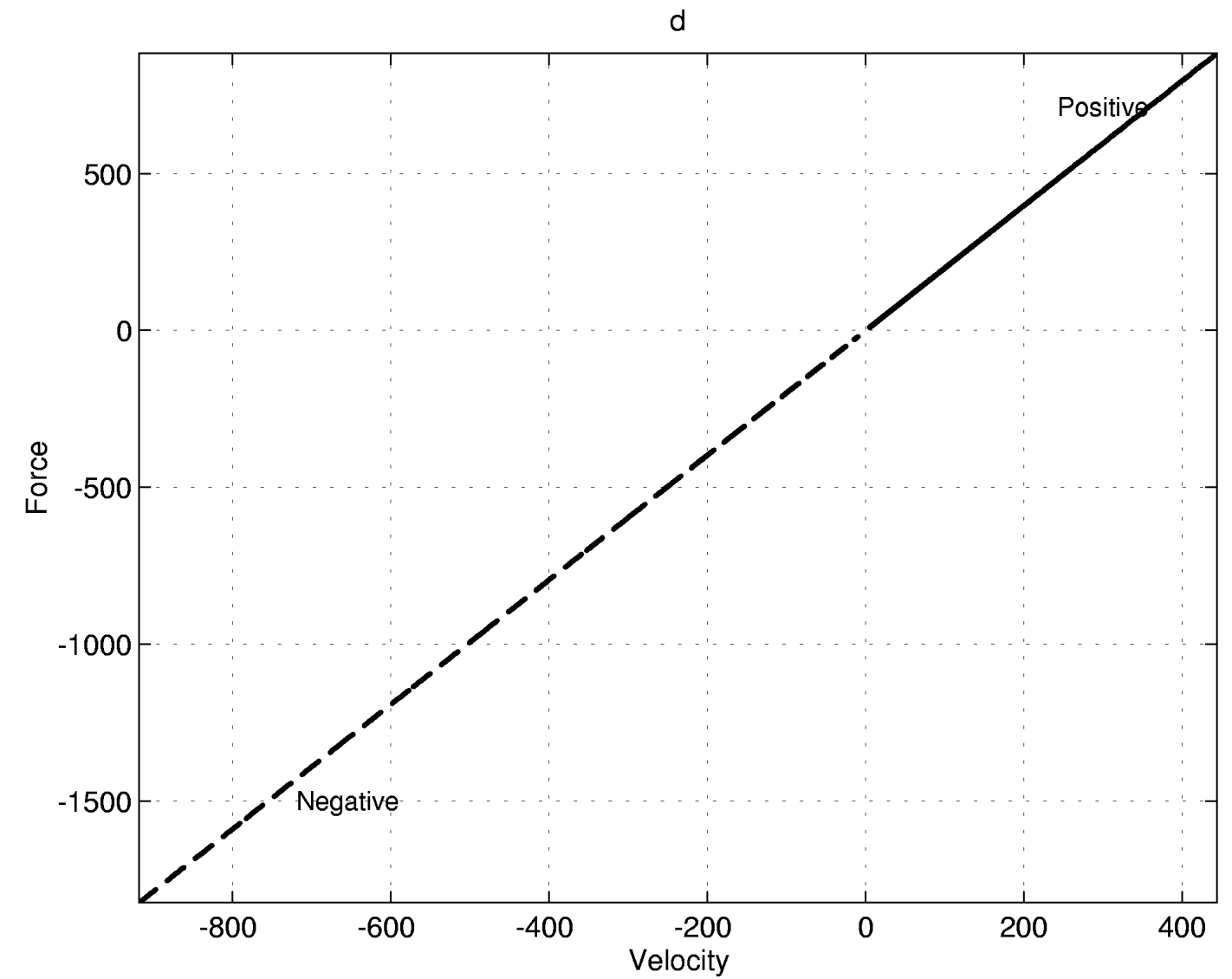

Figure 3d: Estimated force characteristics of bi-linear system - friction force characteristic 


\subsection{Free vibration of non-symmetric system with two cubic like stiffnesses}

The next example is the case of non-symmetric free vibration of two cubic like stiffnesses:

$$
\begin{aligned}
& \ddot{y}+2 \dot{y}+F(y)=0 \\
& F(y)=\left\{\begin{array}{l}
(20 \pi)^{2}\left(1+5 y^{2}\right) y, \text { if } y>0 \\
(40 \pi)^{2}\left(1-3 y^{2}\right) y, \text { if } y \leq 0
\end{array} .\right.
\end{aligned}
$$

Computer simulation, performed for 1024 points with sample frequency $1 \mathrm{~Hz}$, is shown in Figure 4 . The two obtained backbones shown in Figure 5 indicate the varying value of the frequency of the system. The hardening backbone for the positive displacement and the softening backbone for the negative displacement

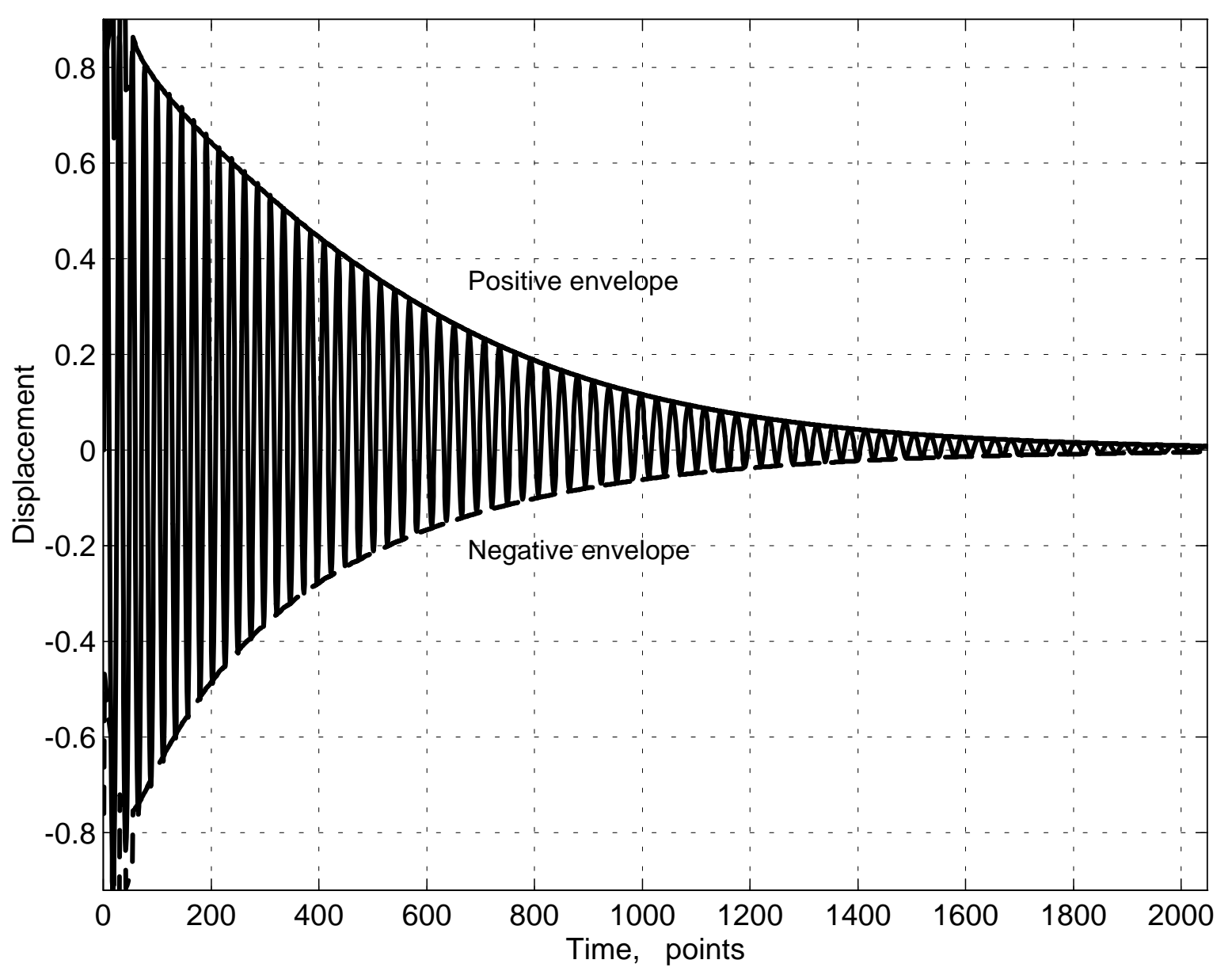

Figure 4: Non-symmetric free vibration of two cubic like stiffnesses 


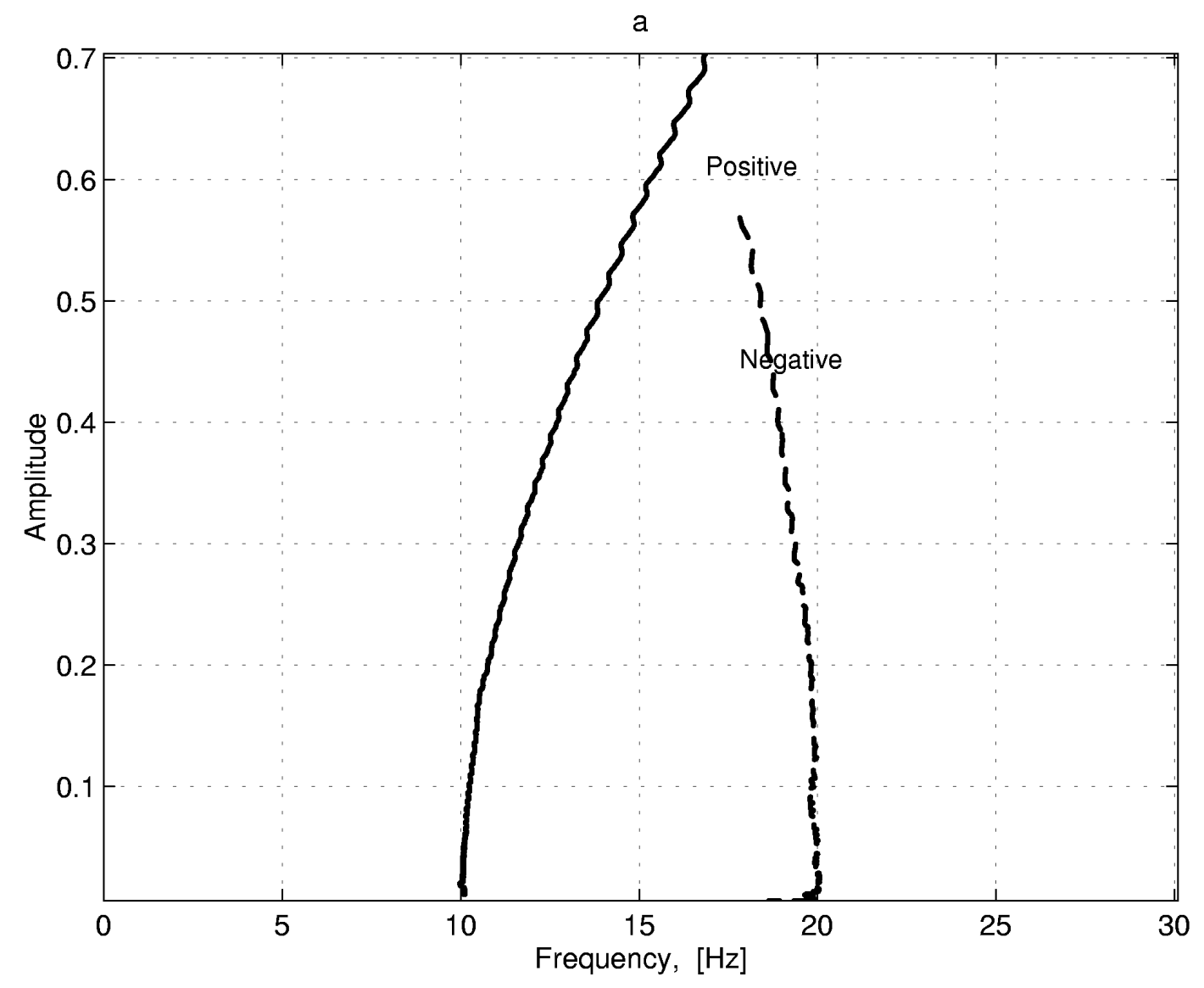

Figure 5a: Estimated force characteristics of two cubic like stiffnesses - backbone

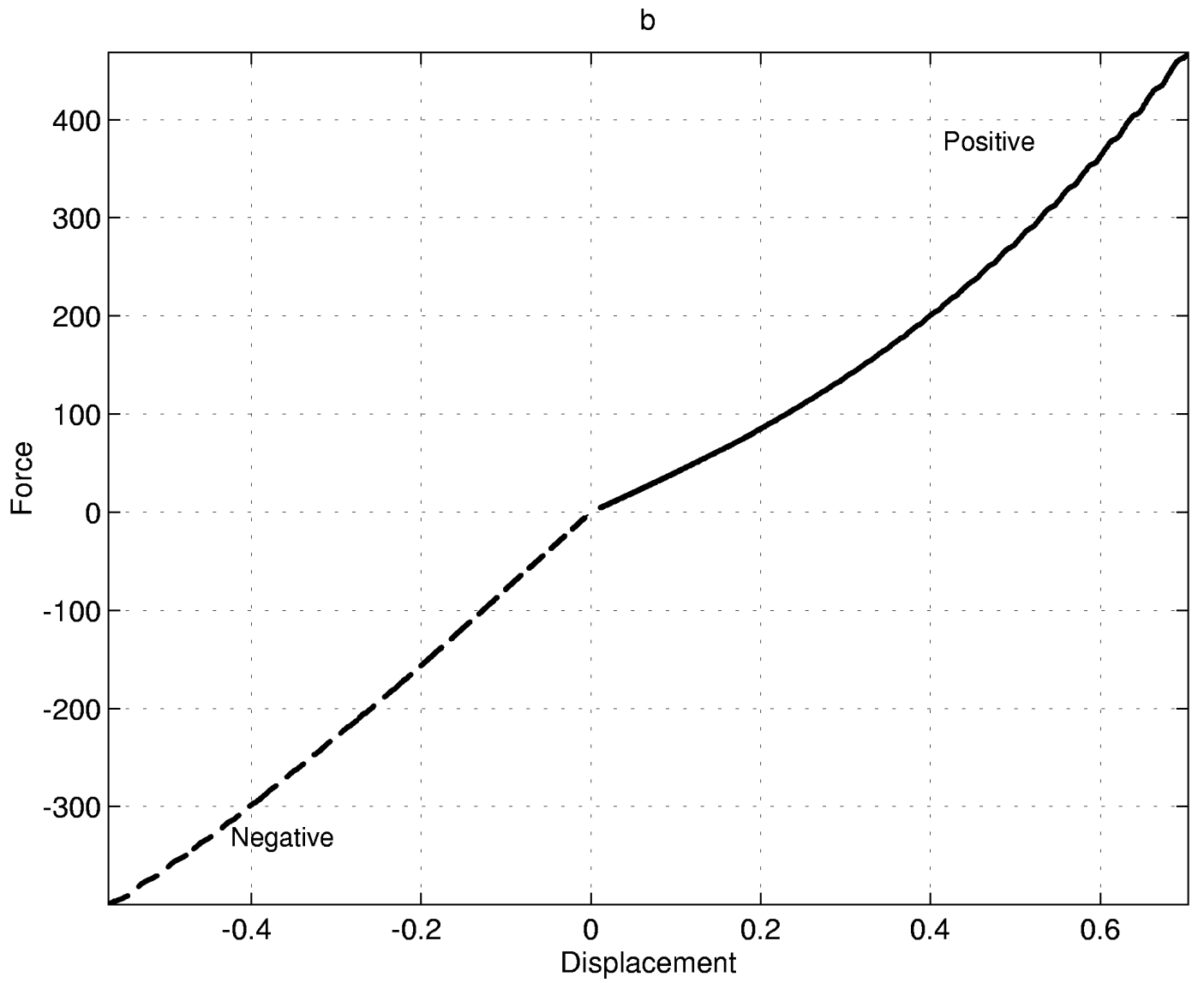

Figure 5b: Estimated force characteristics of two cubic like stiffnesses - spring force characteristic 


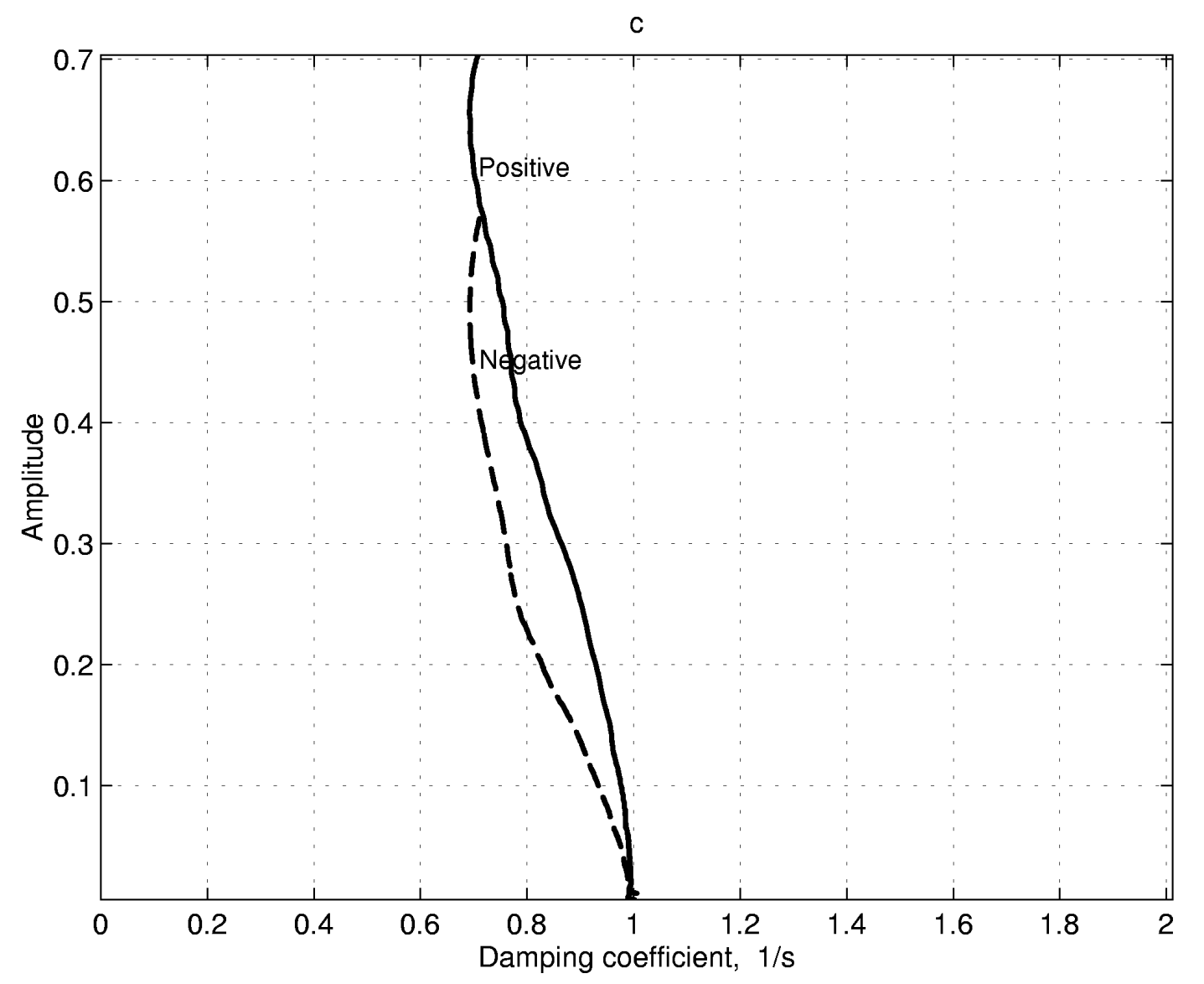

Figure 5c: Estimated force characteristics of two cubic like stiffnesses - damping curve

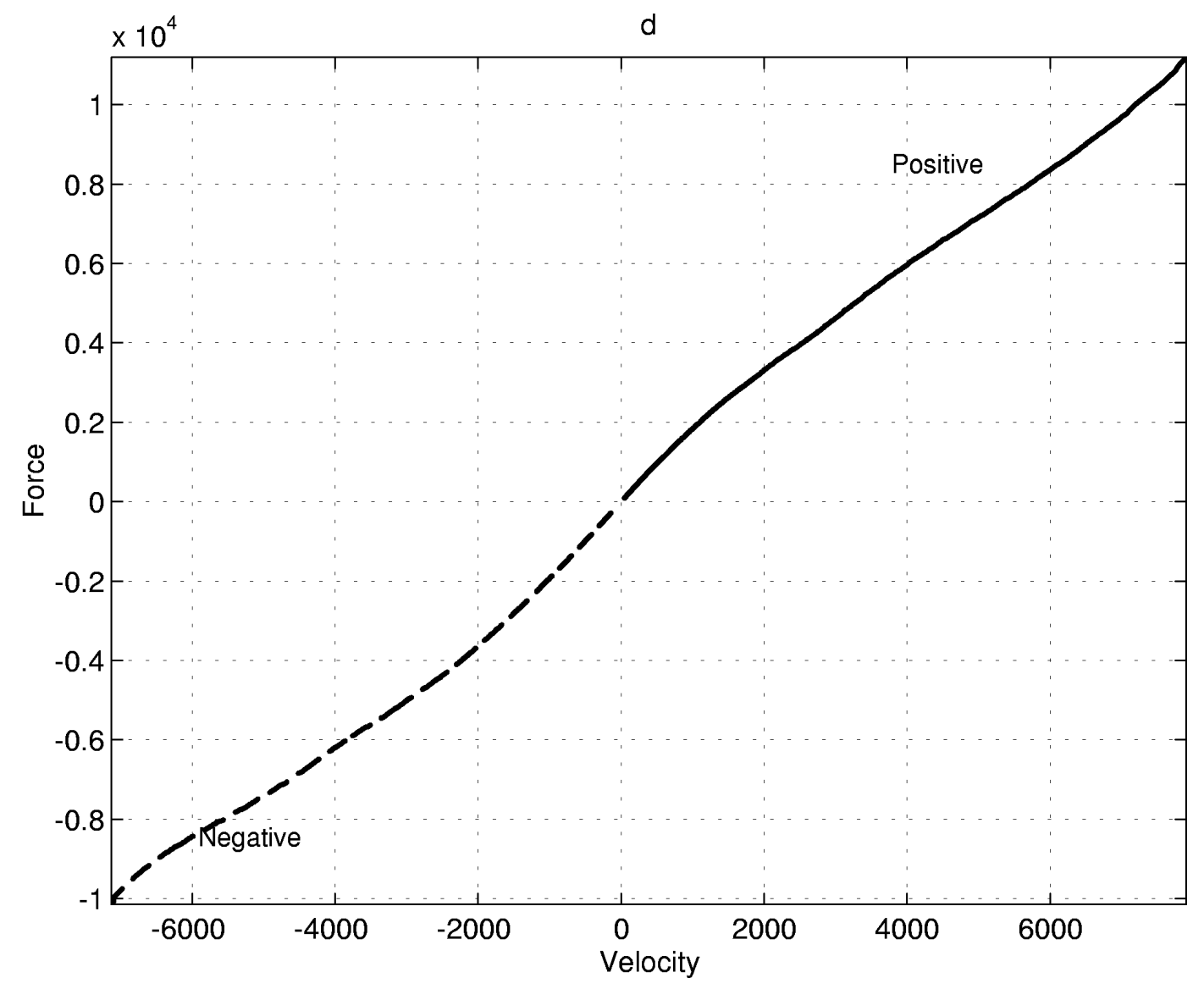

Figure 5d: Estimated force characteristics of two cubic like stiffnesses - friction force characteristic 


\section{THEORETICAL BACKGROUND: DYNAMIC BEHAVIOR OF A ROTOR WITH A CRACKED SHAFT}

\subsection{Diagnosis of cracks in rotating shafts}

Investigations concerning the dynamic behavior of a rotor with a cracked shaft receive increasing interest, especially those aiming at the diagnosis of the damage. The reason is quite obvious: severe damages in power plants have been reported in the last years, many of them due to fatigue cracks in the turbine shaft, Haas (1977), Höxtermann (1988), Muszynska (1992). As a consequence, tools for the monitoring and diagnosis are being developed to avoid such catastrophic failures. The aim is to diagnose cracks at an early level, so that a sound calculation of the remaining life-time can be performed.

The paper by Wauer (1990) summarizes the efforts performed in the field of the modeling and detection of cracks. The first investigations performed were restricted to the basic phenomena occurring due to a cracked rotor shaft. They were based on the assumption of a simple Jeffcott-rotor model, which consists of an elastic massless shaft on hinged supports, with a rigid disc placed in the middle of the shaft. The location of the crack is supposed to be next to the disc, Gasch (1976). The governing phenomenon for the dynamic behavior of a cracked rotor is the "breathing" of the crack, i.e. the opening and closing of the crack during the rotation of the shaft. Generally, the breathing depends on the vibrations of the rotor and therefore on the momentary displacements. Therefore, the system behavior is described by nonlinear differential equations.

\subsection{A finite beam element with a crack - the Theis model}

In real life, the dynamic behavior of turbomachinery is governed by weight dominance: the vibration amplitudes of the rotor are small compared to its static displacement. Then, simplified linear differential equations of motion can be derived, see e.g. Dimarogonas and Papadopoulos (1983), Mayes and Davies (1983). The crack can be assumed as an external load ("crack load"), and the breathing is described as a function of the angle of rotation $\varphi$.

Theis (1990) has developed a finite beam element with a breathing crack which takes into account all six degrees of freedom of the Bernoulli beam theory and is based on the work of Papadopoulus and Dimarogonas (1988). The additional compliance due to the crack is derived based on fracture mechanical considerations via the energy release rate. In contrary to earlier works, the Theis model considers the "semiopen" crack in addition to the "open" and the "closed" crack. The transition between these states is described by heuristic rating functions, and the breathing of the crack depends on the vector of bending moments in the cracked cross section. This crack model requires bending dominance, which means that the breathing only results from the bending vibrations, which in turn influence the lateral and torsional vibrations via cross coupling terms. No additional demands concerning the torsional degrees of freedom are required; however, lateral forces cannot be taken into account. Therefore, the crack model is not valid for critical frequency ranges concerning the lateral vibrations.

The Theis allows to consider the coupling between the bending vibrations and the lateral and torsional vibrations due to the crack. In the following, however, the governing equations are given for bending vibrations only. Furthermore, weight dominance is assumed. Employing the small amplitude approximation, the following linear differential equations can be derived, where the additional dynamics due to the crack are modeled as "external crack loads" acting on the system, Theis (1990), Seibold and Weinert (1996):

$$
\underline{M} \Delta \underline{\ddot{q}}(\varphi)+\underline{D} \Delta \underline{\dot{q}}(\varphi)+\underline{K}_{0} \Delta \underline{q}(\varphi)=\underline{F}_{R}(\varphi)+\underline{F}_{U}(\varphi),
$$

$\underline{M}$ and $\underline{D}$ being the mass and damping matrices, $\underline{F}_{U}$ being the unbalance excitation, and denoting the crack loads as

$$
\underline{F}_{R}=-\Delta \underline{K}(\varphi) \underline{q}_{S} .
$$


In Theis (1990), it is described how the additional compliance due to the crack can be derived via the energy release rate based on fracture mechanical considerations. In order to facilitate the application to model-based damage diagnosis, the crack loads can be approximated by polynomial functions, Seibold (1995):

$$
\underline{F}_{R}(a, \varphi)=\left(\sum_{i=0}^{n} a^{i} \underline{P}_{i}\right) \underline{\gamma}(\varphi)=\underline{R}(a) \underline{\gamma}(\varphi),
$$

where a is the depth of the crack, $\underline{P}_{i}$ are constant matrices of coefficients independent of a and $\varphi$, and

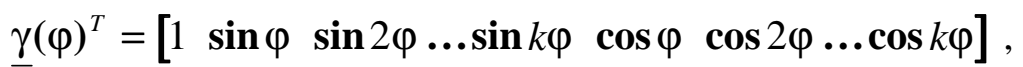

is a function of the angle of rotation $\varphi$.

The Theis model does not require weight dominance, and it can be modified for non-constant speeds of rotation. Still, one quite severe restriction needs to be mentioned: the model is only valid for cracks with a depth less than the radius of the shaft. However, the modified Theis-model according to equations (13-14) facilitates the application to damage diagnosis. Based on the concept of modeling the crack as "external loads", the crack element can be easily implemented in existing FE programs. Figure 6 shows the crack model: the crack, which need not be located in the middle of the beam element, is mapped as external forces and moments $X_{i}$. This crack element then can be "added" to a FE model of a rotor, Figure 7.

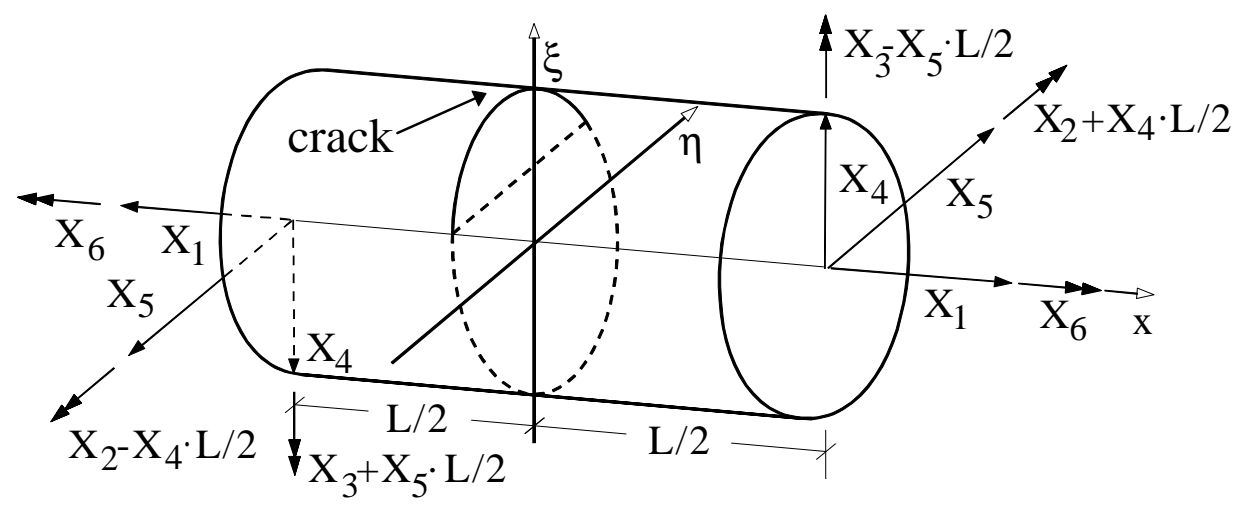

Figure 6: Crack model of Theis.

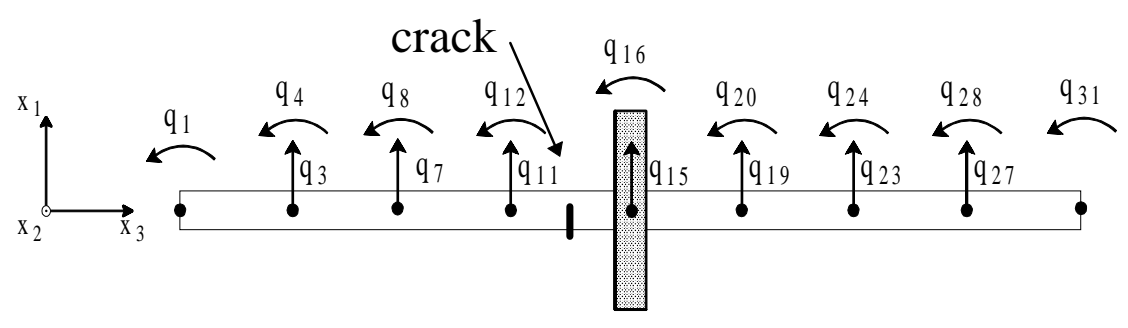

$\begin{array}{llllllllll}\text { nodes } & 1 & 2 & 3 & 4 & 5 & 6 & 7 & 8 & 9\end{array}$

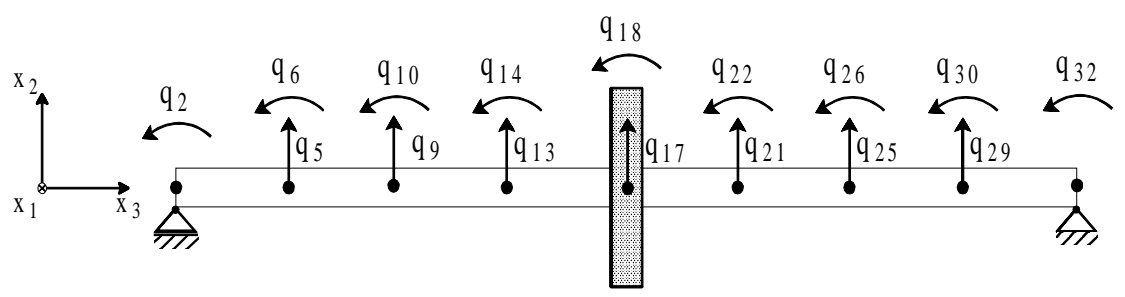

Figure 7: Finite-Element model of a rotor with a cracked shaft. 


\section{MULTI-HYPOTHESIS TESTING WITH A BANK OF KALMAN FILTERS}

Multi-hypothesis testing based on a bank of Kalman filters is a powerful tool for the detection of the location of damage. The theoretical background and an application to cracks in rotating shafts is described in Seibold and Weinert (1996). This model-based approach requires a suitable model of the system, as for instance the FE model shown in Figure 7. The idea is to design different Kalman filters for different damage hypotheses (bank of filters, Figure 8). In our case, supposing we want to detect the location of a crack in a rotor modeled according to Figure 7, nine different hypotheses are needed: the zero-hypothesis based on a FE model of the rotor without a crack, and eight hypotheses based on FE models of the rotor with a crack in beam elements 1-8. Each Kalman filter generates innovations $\underline{v}$ for every time step $k$ :

$$
\underline{v}_{k+1}=\underline{y}_{k+1}-\underline{C}_{\hat{z}_{k+1 / k}}
$$

where $\underline{y}_{k+1}$ - the vector of measurements at time step $k+1, \underline{\underline{z}}_{k+1 / k}$ - the model prediction for the time step $k+1$, and $\underline{C}$ - the measurement matrix. These innovations are the differences between model prediction and measurements. Usually, the measurement noise is normally distributed and uncorrelated white noise, so that the innovations of Kalman filters are time series with the same properties if measurement and model prediction are corresponding. Therefore, by statistically analyzing the innovations of each Kalman filter in the bank of filters, the location of the crack can be determined. Furthermore, if Extended Kalman Filters (EKF) are employed, a parallel state and parameter estimation can be performed, and the depth of the crack can be calculated, too.

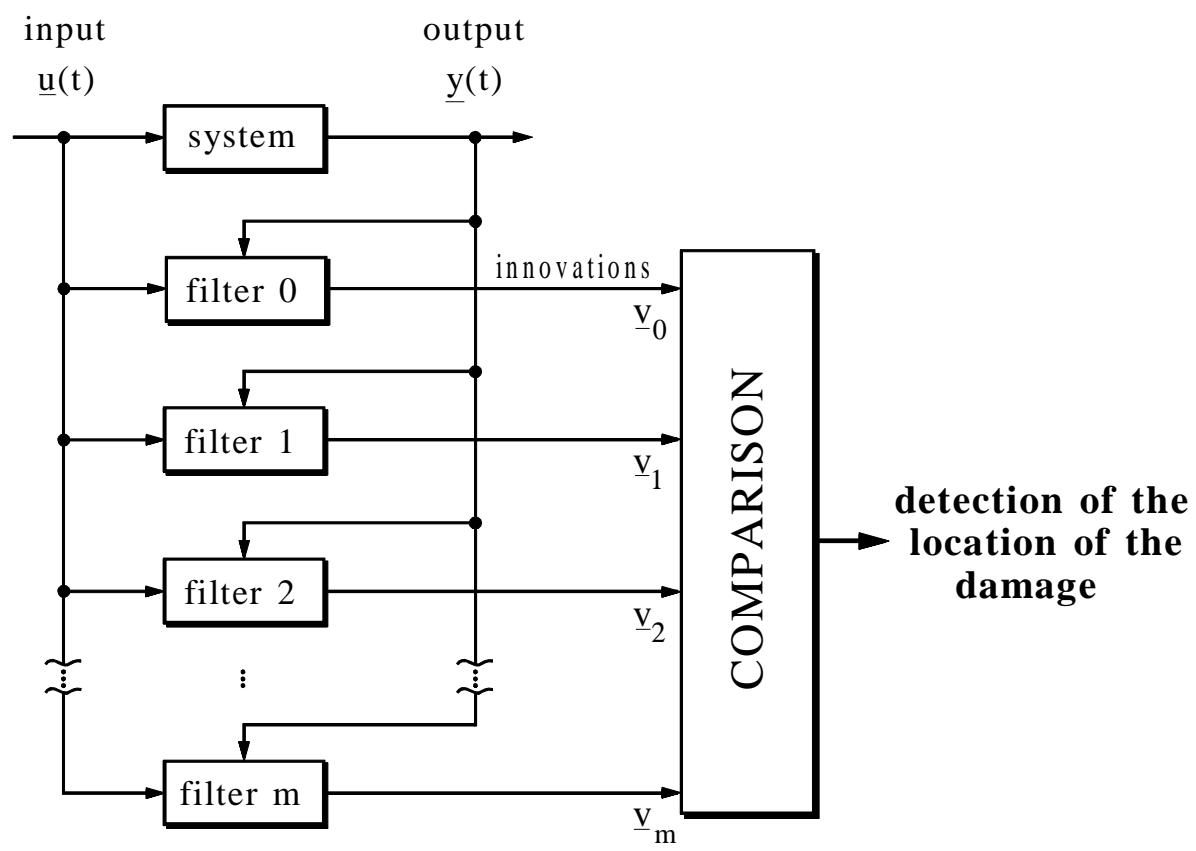

Figure 8: Bank of filters. 


\section{ROTOR TEST RIG: IDENTIFICATION OF A CRACK IN THE ROTOR SHAFT}

A simple rotor test rig was built in order to apply the proposed procedure to real measurements, Figure 9. The test rig consists of a shaft with radius $\mathrm{R}=9 \mathrm{~mm}$ on hinged supports. A disc is mounted in the middle. Initiated by a notch of about $2 \mathrm{~mm}$ depth, a transverse crack in the shaft at a location close to the disc is introduced. Two kinds of vibrations are measured: free vibrations of the disc initiated by an impulse, and stationary vibrations at constant speed. Then, static overloads are applied using a special apparatus. This results in dark lines (beachmarks) on the crack face. After the experiment, the measurements taken can be related to the beachmarks and to the actual crack depths. In this way, the results of the identification can be checked. The experimental set-up is described in detail in Seibold (1995).

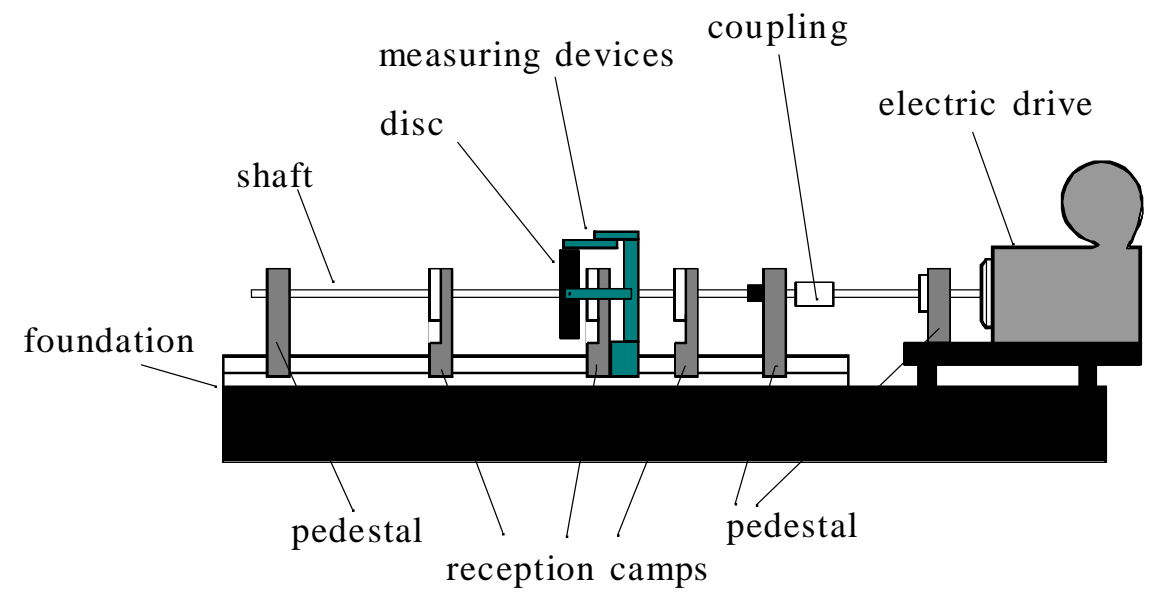

Figure 9: Rotor test rig.

\subsection{Detection of the crack with the HT approach}

An experimental identification of the rotor structure with a notch and crack was made on the basis of 4 separate measurements (512 time steps) of free vibrations of the disk (crack at the lowest position). In this experiment, the depth of the crack was $5 \mathrm{~mm}$. Free vibrations were picked up from the non-rotating rotor after exciting the system with an impulse hammer. The proposed HT method enables us to separate the measured non-symmetric vibration into two different parts. The first part (Figure 10, bold line) describes system behavior for only positive displacement, and the second part (Figure 10, dashed line) for only negative displacement. The obtained results of the HT identification, shown in Figure 10, indicate the closely-spaced positive and the corresponding negative backbones of the 4 separate measurements. The tested system has a strong non-symmetric elastic force characteristic: the positive movement exhibits smaller values of the natural frequency $(25.5-26 \mathrm{~Hz})$. The difference between the natural frequencies for the positive and the negative movement is about $2 \mathrm{~Hz}$.

The system also has a small non-linear elastic force characteristic, dissimilar for the positive and the negative movement. The positive movement back-bone looks like a hardening non-linear spring. On the contrary, the negative movement back-bone shows small softening non-linear spring characteristics. The tested system has a symmetric friction characteristic. The estimated friction force characteristics possess a strong dry friction nature. 


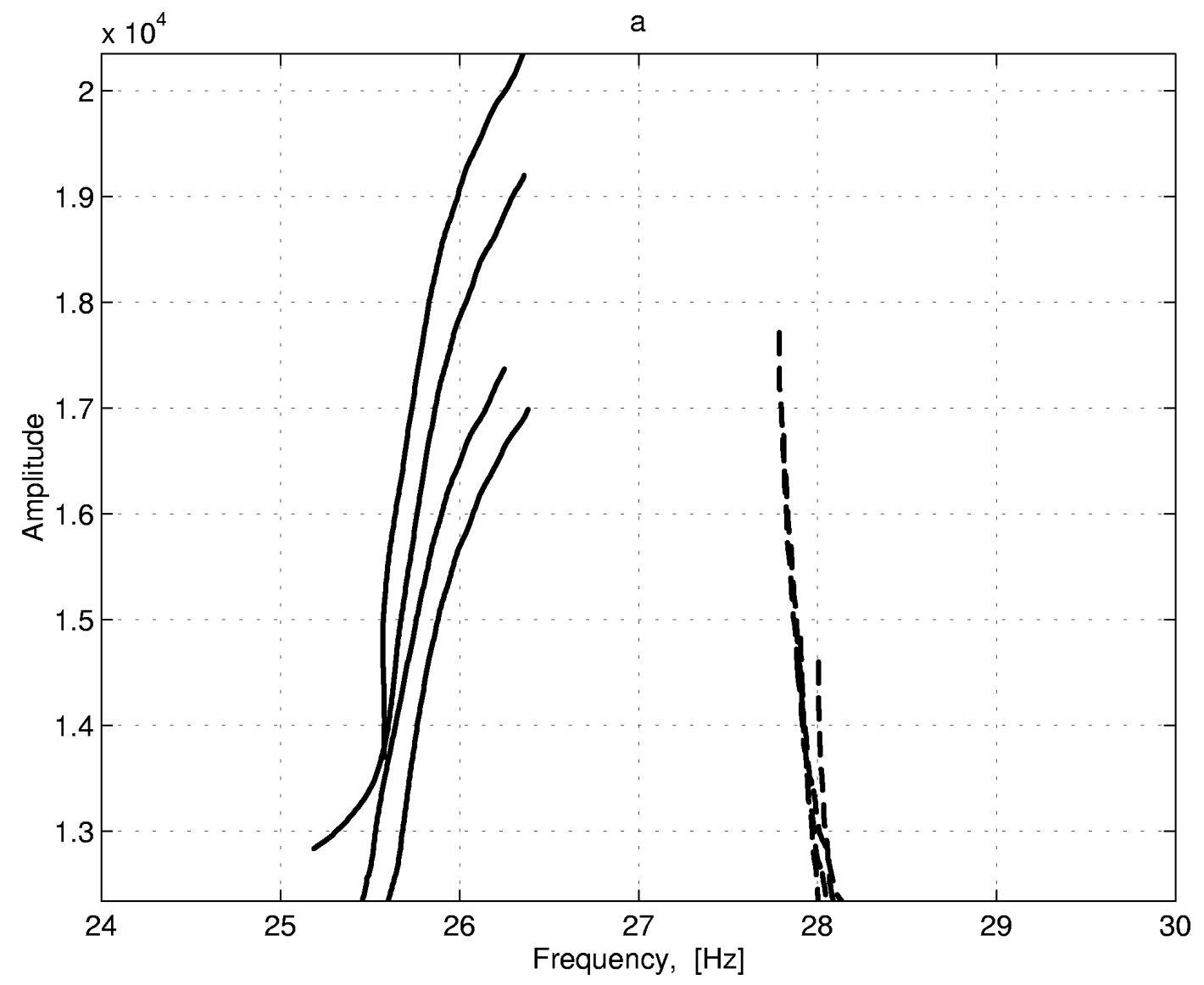

Figure 10a: Estimated force characteristics of cracked structure - backbone

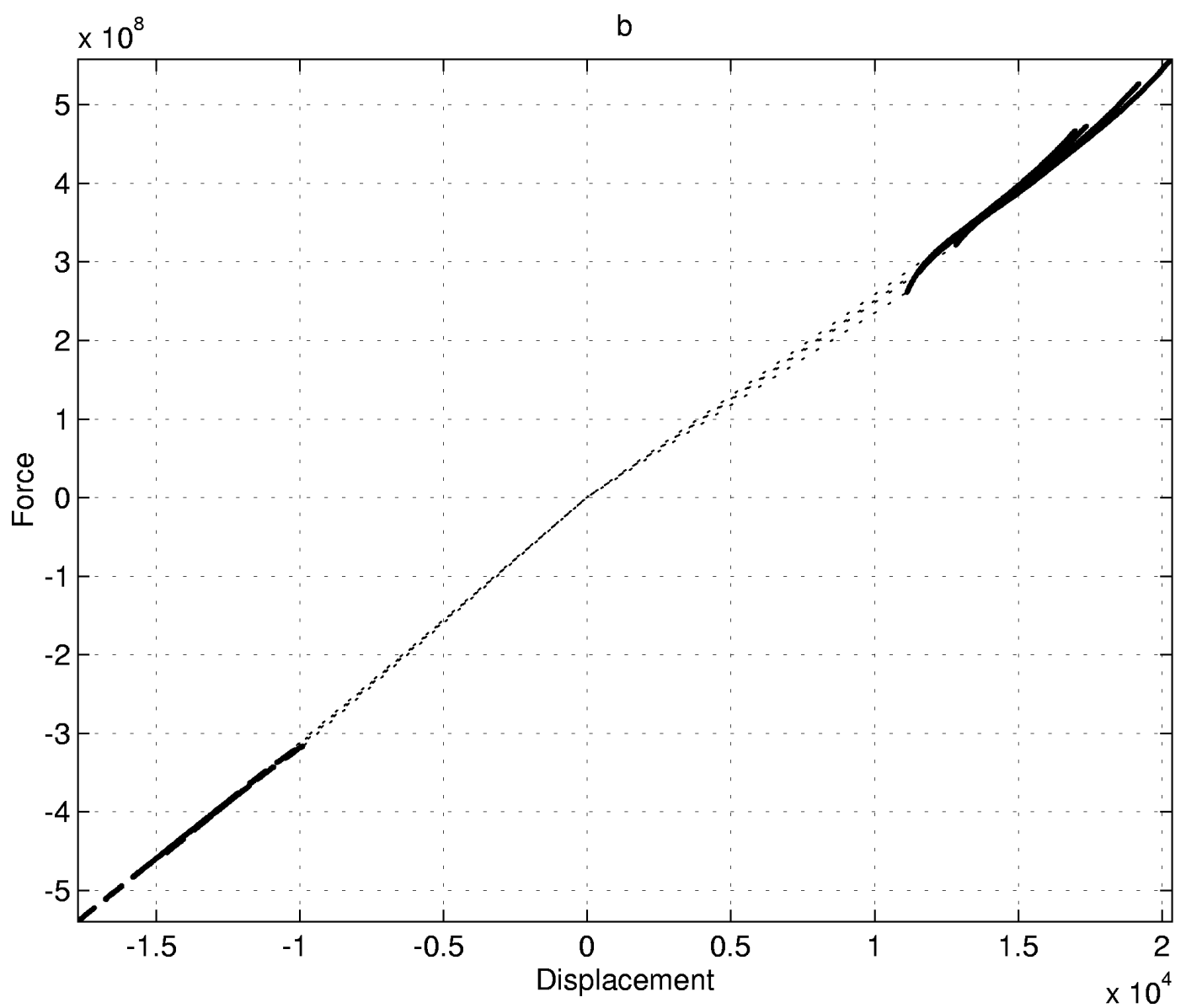

Figure 10b: Estimated force characteristics of cracked structure - spring force characteristic 


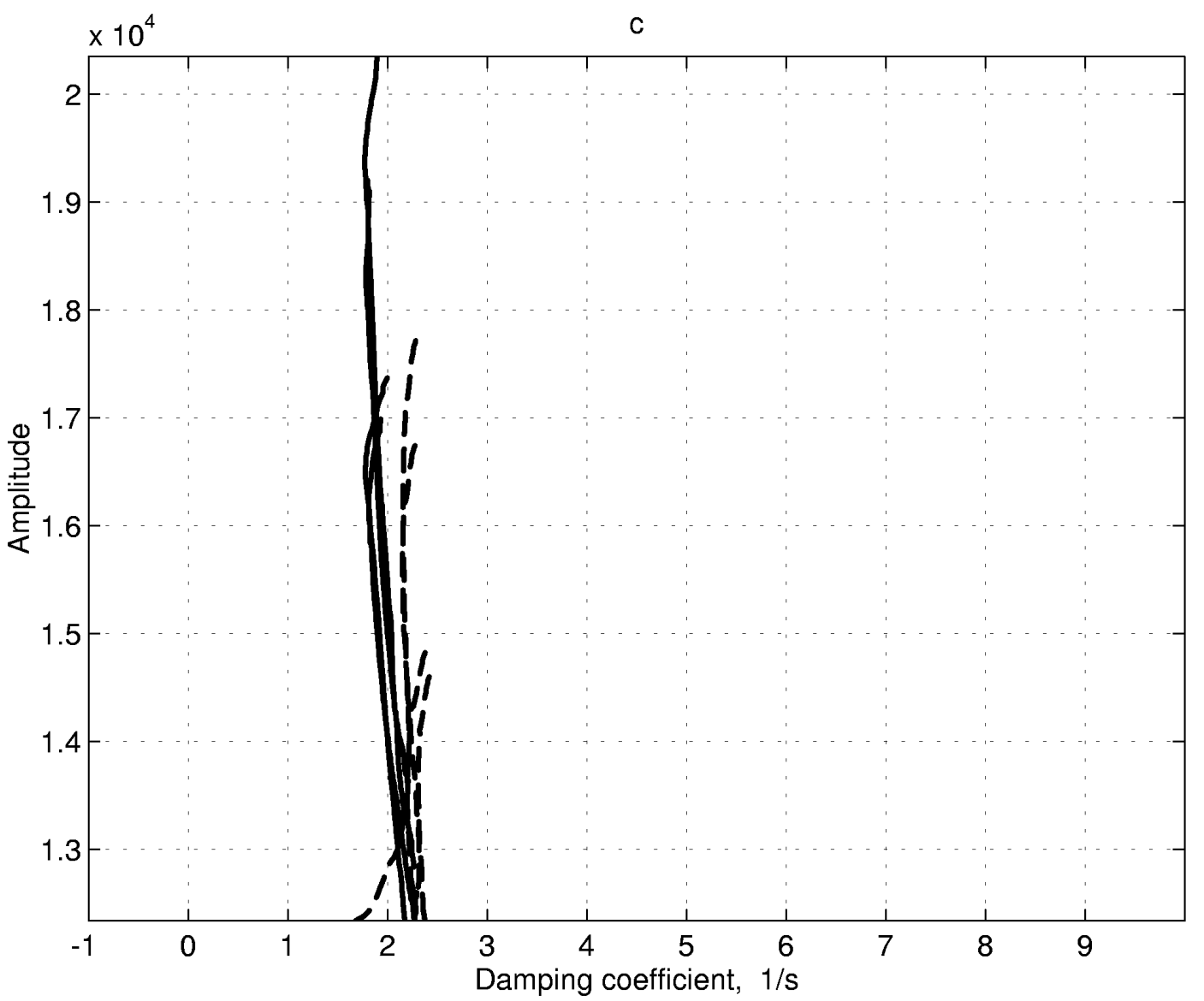

Figure 10c: Estimated force characteristics of cracked structure - damping curve

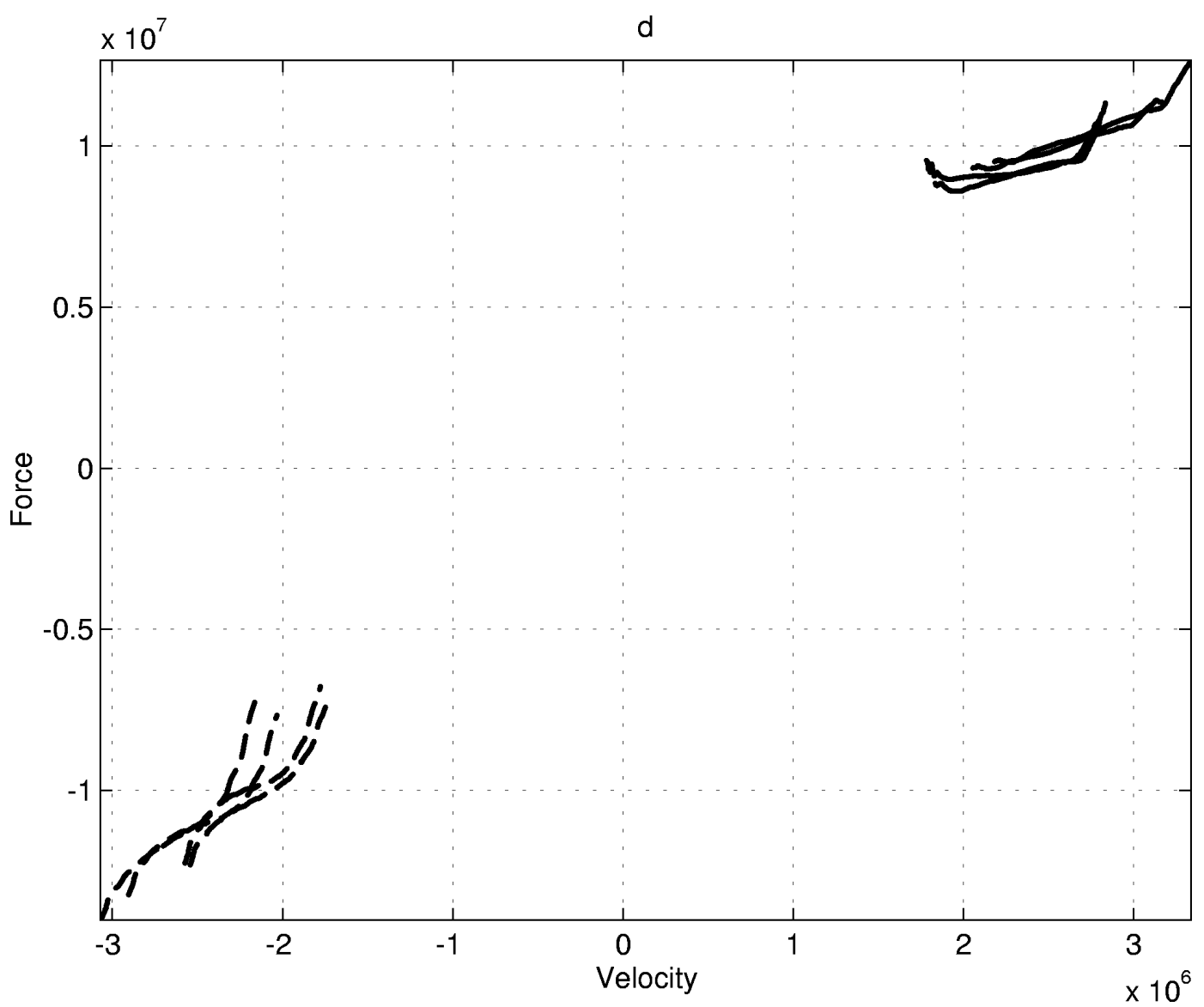

Figure 10c: Estimated force characteristics of cracked structure - friction force characteristic 


\subsection{Detection of the location of the crack with multi-hypothesis approach}

The results of the previous section clearly indicate the presence of a crack. Now, the location and depth of this crack can to be determined employing the multi-hypothesis approach described in Section 5, and the measured vibrations of the disk at constant speed. Considering our model of the rotor, Figure 7, 9 damage hypotheses and therefore a bank of nine EKFs are needed. One filter is based on the null hypothesis "no crack", and eight filters have to be designed based on the hypothesis "crack in beam element no.i". These eight filters are based on a finite element model of the rotor with a crack in the specific beam element, and perform a parallel state and parameter estimation, where the parameter to be estimated is the depth of the crack. In order to avoid excessive computation time, a modal condensation to the first four bending eigenforms is performed. Without the initial information about the type of the damage, many more damage hypotheses (e.g. increasing unbalance) would have to be considered, so that the approach would be a lot more time-consuming.

Figure 11 shows the identification of the crack depth $a$ based on the true hypothesis no. 4 . The true crack depth is $5 \mathrm{~mm}$, and the identified value is $5.2 \mathrm{~mm}$. The parameter converges quickly. Figure 12 shows the mean of the related standard deviations of the different damage hypotheses. If the detection of the location is successful, the correct hypothesis should possess the smallest standard deviation, Mehra and Peschon (1971).

As has been stated above, the measurement set-up only allows a measurement of the displacements of the disc. It is not possible to pick up measurements next to the pedestals, due to the diameter of the shaft, which is relatively small. Therefore, it is impossible to determine whether the crack is on the left-hand or righthand side of the disc, and symmetric hypotheses like 1 and 8,2 and 7, 3 and 6, 4 and 5 possess the same standard deviations. Still, it is obvious from Figure 12 that the crack is in one of the beam elements next to the disc, i.e. beam element no. 4 or 5 .

The computations were performed using PC-Matlab. For the processing of 2000 time steps, each filter in the bank needs less than 30 seconds computation time.

In Seibold and Weinert (1996) it is shown that the crack location can be determined very well if more sensors are available. Of course, a bank of Kalman Filters may also be designed on the basis of a nonlinear model of the cracked rotor. In Seibold $(1995,1997)$, it was shown that the crack depth can be identified based on a nonlinear Jeffcott-rotor model. Furthermore, smaller cracks can be diagnosed very well, Seibold (1995, 1997). It is possible, too, to consider nonconstant speeds of rotation.

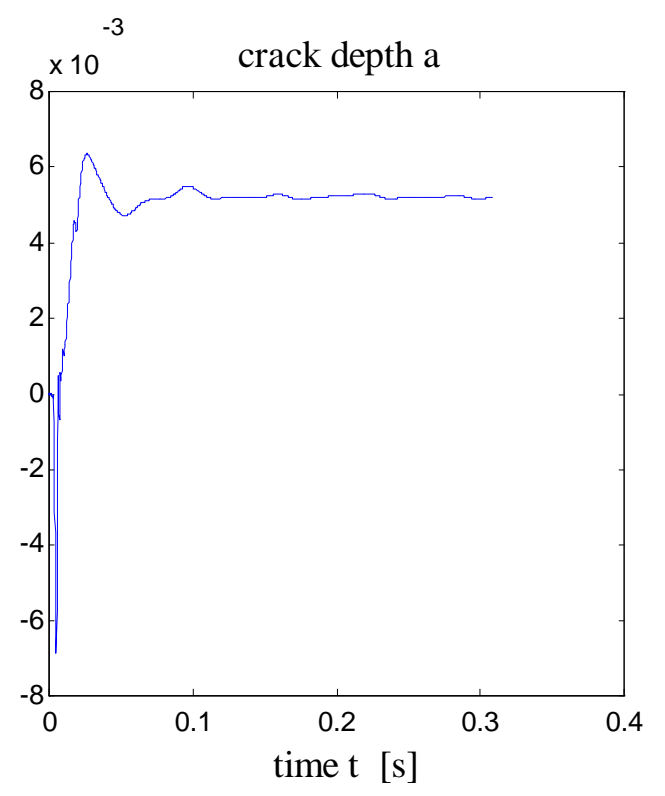

Figure 11: Identification of the crack depth a; hypothesis no. 4. 


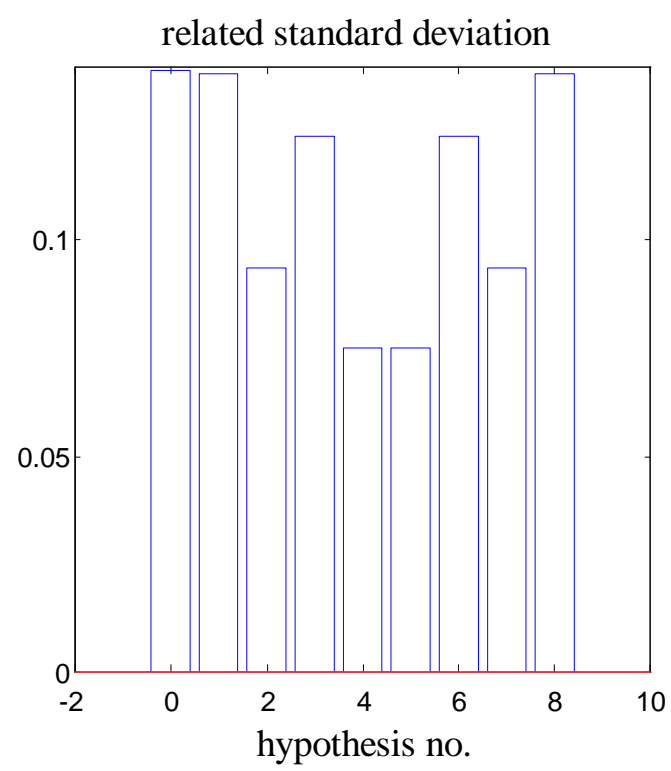

Figure 12: Related standard deviations (mean) of the different hypotheses. 


\section{COMPARISON TO OTHER DIAGNOSTIC PROCEDURES AND CONCLUSION}

Recently, the problem of damage detection and diagnosis is tackled by many researchers from different fields. Most of the approaches are based on the investigation of modal quantities, like changes in eigenfrequencies and eigenforms. A recent paper showed that the size and location of a crack in a rotor can be calculated by investigating the changes in the first four harmonics, Hachmann and Popp (1997). The approach is very interesting and validated by measurements taken at a rotor test rig. However, the authors state that the eigenfrequencies have to be determined very accurately, which is in practice not always possible. Furthermore, small cracks have only insignificant influences on the eigenfrequencies, so that they cannot be detected by monitoring the eigenfrequencies only.

A completely different approach is suggested by Fritzen, Jennewein and Buchen (1996). They propose to employ a model representing the undamaged vibrating structure and a local description of the damage, and show that multiple cracks in a steel frame as well as a "lost mass" in a spatial structure can be identified quite accurately. Up to now, only non-rotating structures were treated.

Damage detection was the main subject of the last IMAC conference, IMAC XV (1997). The paper by Worden (1997) describes how Artificial Neural Networks can be employed for condition monitoring, and an application to a simulated 3-dof system is treated. Recently, the research in this area is very often associated with methods of novelty or anomaly detection, very similar to the idea of multi-hypotheses testing. This can also be stated about the approach presented by Abdelghani, Basseville, and Beneviste (1997), where damage is diagnosed by comparison to a reference model. For each hypothesized damage, a test statistics is generated, and the statistics measure the likelihood of the most likely change (i.e. damage) in the structure.

According to the knowledge of the authors, there is no combined approach documented in the literature as the one presented in this paper, except for a paper published by one of the authors, Bucher and Seibold (!997). The modal procedures mentioned above, as well as the purely signal-based procedures in general yield excellent results concerning the detection of damage, but, usually, their drawback is the inherent difficulty to determine the location and extent of damage. This gap can be overcome very well by a modelbased approach, which on the other hand is quite time-consuming and usually not applicable for on-line monitoring. Furthermore, model-based procedures might sometimes have difficulties to distinguish between specific damages, like a crack and an increasing unbalance. Therefore, a combination of the two methodologies will yield an improved detectability of damages at early stages compared to using each approach separately. However, it is not possible to quantify this improved detectability. Generally, the success of a signal-based approach depends very much on the initial physical knowledge of the user. It may yield valuable information about the type of the damage, but its extent of a damage can only very rarely be calculated. A purely model-based approach, on the other hand, can fail if no initial knowledge about the damage is available.

The authors feel that a true progress in the area of diagnostics can only be made if signal- and model-based diagnosis are combined, as proposed in this paper, where the applicability to the diagnosis of cracks in rotors was proven. In the first simulated example, the application of the HT for the decomposition of nonsymmetric signals was shown. Then, the combined methodology was applied to a rotor test rig with a cracked shaft. The results of the HT clearly indicated the type of damage: a crack in the rotor shaft, so that subsequently a bank of Kalman Filters based on a suitable model of the damage could be designed. By multi-hypothesis testing, the location and the depth of the crack could be determined.

Further research will be devoted to investigate the applicability to smaller cracks, as well as to other types of damages.

\section{ACKNOWLEDGEMENT}

The project is partly financed by the German Research Association (DFG) under contract no. Se 578/5-1 which is highly appreciated. 


\section{REFERENCES}

Abdelghani, M., Basseville, M. Beneviste, A., 1997, "In-operation damage monitoring and diagnostics of vibrating structures, with application to off-shore structures and rotating machinery", Proc. 15th IMAC, International Modal Analysis Conference Vol. II, February, Orlando, FL, USA, 1815-1830.

Bucher, I. and Seibold, S, 1997, "Using combined temporal and spatial information in multi-hypothesis based damage detection for rotating machines“, Proc. 15th IMAC, International Modal Analysis Conference Vol. I, February, Orlando, FL, USA, 870-876.

Dimarogonas, A.D. and Papadopoulos, C.A., 1983, "Vibration of cracked shafts in bending", Journal of Sound and Vibration 91 (4), 583-593.

Feldman, M.S., 1985, "Investigation of the natural vibrations of machine elements using the Hilbert transform", Soviet Machine Science, Allerton Press Inc. \# 2, 44-47.

Feldman, M., 1994a, "Non-linear system vibration analysis using Hilbert transform -- I. Free vibration analysis method "FREEVIB"'", Mechanical Systems and Signal Processing 8(2), 119-127.

Feldman, M., 1994b, "Non-linear system vibration analysis using Hilbert transform -- II. Forced vibration analysis method "FORCEVIB"", Mechanical Systems and Signal Processing 8(3), 309-318.

Feldman, M., 1997, "Vibration analysis of non-symmetric elastic force systems via the Hilbert transform", Proc. 15th IMAC, International Modal Analysis Conference Vol. I, February, Orlando, FL, USA, 10171022.

Fritzen, C.-P., Jennewein, D. and Buchen, D., 1996, "Model based damage detection from vibration data", Proc. ISMA 21, International Conference on Noise and Vibration Engineering, P. Sas (ed.), Leuven 10171031.

Gasch, R., 1976, "Dynamic behaviour of a simple rotor with a cross-sectional crack", Conference on Vibrations in Rotating Machinery, IMechE Conference Publications, University of Cambridge.

Haas, H., 1977, "Großschäden durch Turbinen- oder Generatorläufer, entstanden im Bereich bis zur Schleuderdrehzahl", Der Maschinenschaden 50, Heft 6, 195-204, in German.

Hachmann, I., and Popp, K., 1997, "Ermittlung des Rißortes angerissener Wellen im ruhenden und rotierenden System", SIRM'97 Schwingungen in Rotierenden Maschinen IV, Irretier, Nordmann, Springer (eds.), Vieweg Verlag, 191-198.

Höxtermann, E., 1988, "Erfahrungen mit Schäden in Form von Anrissen und Brüchen an Dampfturbinenwellen, Radscheiben und Generatorläufern", VGB Technisch-Wissenschaftliche Berichte Wärmekraftwerke - VGB-TW 107, in German.

IMAC XV, 1997, Proc. 15th IMAC, International Modal Analysis Conference, February, Orlando, FL, USA.

Mayes, I.W. and Davies, W.G.R., 1983, "Analysis of the response of a multi-rotor-bearing system containing a transverse crack in a rotor", ASME Design Engineering Technical Conference, Dearborn, MI, Paper 83-DET-84.

Mehra, R.K. and Peschon, J., 1971, "An innovations approach to fault detection in dynamic systems", Automatica 7, 637-640.

Mitra, S.K. and Kaiser, J.F., 1993, Handbook for Digital Signal Processing, Wiley-Interscience, 1268.

Muszynka, A., 1992, "Vibrational diagnostics of rotating machinery malfunctions", Course on Rotor Dynamics and Vibration in Turbomachinery, von Karman Institute for Fluid Dynamics, Belgium.

Papadopoulos, C.A. and Dimarogonas, A.D. 1988, "Coupled longitudinal and bending vibrations of a cracked shaft“, Journal of Vibration, Acoustics, Stress and Reliability in Design Vol. 110, 1-8.

Seibold, S., 1995, Ein Beitrag zur modellgestützten Schadendiagnose bei rotierenden Maschinen ,VDIFortschrittberichte, VDI-Verlag, 11/219, Düsseldorf. In German. 
Seibold, S. and Weinert, K., 1996, "A time domain method for the localization of cracks in rotors", Journal of Sound and Vibration 195(1), 57-73.

Seibold, S., 1997, "Identification of physical parameters employing an instrumental variables technique", accepted for publication in Mechanical Systems and Signal Processing.

Theis, W., 1990, Längs- und Torsionsschwingungen bei quer angerissenen Rotoren - Untersuchungen auf der Grundlage eines Rißmodells mit 6 Balkenfreiheitsgraden, VDI-Verlag, Düsseldorf, Reihe 11, Nr. 131. In German.

Wauer, J., 1990, "On the dynamics of cracked rotors: A literature Survey", Applied Mechanics Review 43, 13-17.

Williams, J.H. and Davies, A., 1992, "System condition monitoring - An overview", Noise and Vibration Worldwide 23 (9), 25-29.

Worden, K., 1997, "Damage detection using a novelty measure", Proc. 15th IMAC, International Modal Analysis Conference Vol. I, February, Orlando, FL, USA, 631-637.

\section{LIST OF SYMBOLS AND ABBREVIATIONS}

$\begin{array}{ll}\text { HT } & \text { Hilbert Transform } \\ y & \text { system solution } \\ h & \text { damping coefficient } \\ F(y) & \text { non-linear non-symmetrical spring force } \\ A(t) & \text { envelope (instantaneous amplitude) } \\ \psi(t) & \text { instantaneous phase } \\ \dot{\psi}(t)=\omega(t) & \text { instantaneous frequency } \\ \omega_{0}(t) & \text { instantaneous undamped natural frequency } \\ a & \text { depth of the crack } \\ \text { EKF } & \text { Extended Kalman Filter }\end{array}$

